\title{
On estimation the relative risk of small area and visualization spatio-temporal map
}

\author{
Xiaoxiao Song 1,2 , Yan Li 3,2 , Le cai 4,2 , Wei Liu4 4,2 , Wenlong Cui 4,2 \\ ${ }^{1}$ Department of epidemiology and statistics School of Public Health, Kunming Medical university, Kunming, Yunnan Province, China, ${ }^{2}$ Yunnan \\ Provincial Collaborative Innovation Center for Public Health and Disease Prevention and Control, Kunming, China, ${ }^{3}$ Kunming Medical University, \\ Kunming, China, ${ }^{4}$ School of Public Health, Kunming Medical University, Kunming, China
}

\section{Objective}

The purpose is to propose a serial of approach for estimation for disease risk for ILI in "small area" and present the risk values by spatio-temporal disease mapping or an interactive visualization with HTML format.

\section{Introduction}

Disease mapping is a method used to descript the geographical variation in risk (heterogeneity of risk) and to provide the potential reason (factors or confounders) to explain the distribution. Possibly the most famous uses of disease mapping in epidemiology were the studies by John Snow of the cholera epidemics in London. Accurate estimation relative risk of small areas such as mortality and morbidity, by different age, ethnic group, interval and regions, is important for government agencies to identify hazards and mitigate disease burden. Recently, as the innovative algorithms and the available software, more and more disease risk index has been pouring out. This abstract will provide several estimation risk index, from raw incidence to model-based relative risks, and use visual approach to display them.

\section{Methods}

All the data are from a syndromic surveillance and real-time early warning system in the Yunnan province in the China. For brief introduction aim, we are using the ILI (Influenza-like illness) data in December 2017 in one county. The relative risks of disease in small area are including: raw incidence, a standardized morbidity ratio (SMR), Empirical Bayes smoothing estimation relative risk (EB-RR) and the Besag-York-Mollio model (BYM). The incidence in each small area is common used for descriptive the risk but fail to comparable directly since the different population at risk in each area. SMR is a good way to deal with this incomparability. But SMR can give rise to imprecisely estimate in areas with small populations. Empirical Bayes estimation approach has been used for smoothing purpose and can be seen as a compromise between relative risks and $P$-values. However, all above approaches are inept to have spatial or spatio-temporal structure in mind. BYM based the Bayesian inference can handle both the area-specific spatial structured component (such as intrinsic conditional autoregressive component) and the exchangeable random effect (unstructured component). All the analyses are implemented in the R software with INLA package (http://www.rinla.org). The outcome of relative risk estimation with visual way and interactive maps showing are using ggplot 2 and leaflet packages.

\section{Results}

1. the spatio-temporal raw cases of ILI from 2017/12/01 to 2017/12/31 is Fig.1

2. the SMR and EB-RR estimation RR of ILI are in Fig.2 and Fig.3

3. the most excited is the interactive visualization with HTML format for all the risk indexes is visited http://rpubs.com/ynsxx/424814 in detail. And the screenshot is Fig.4

\section{Conclusions}

Small area disease risk estimation is important for disease prevention and control. The faster function of computer with power $\mathrm{R}$ software can lead to advance in disease mapping, allowing for complex spatio-temporal models and communicate the results with visualization way. 


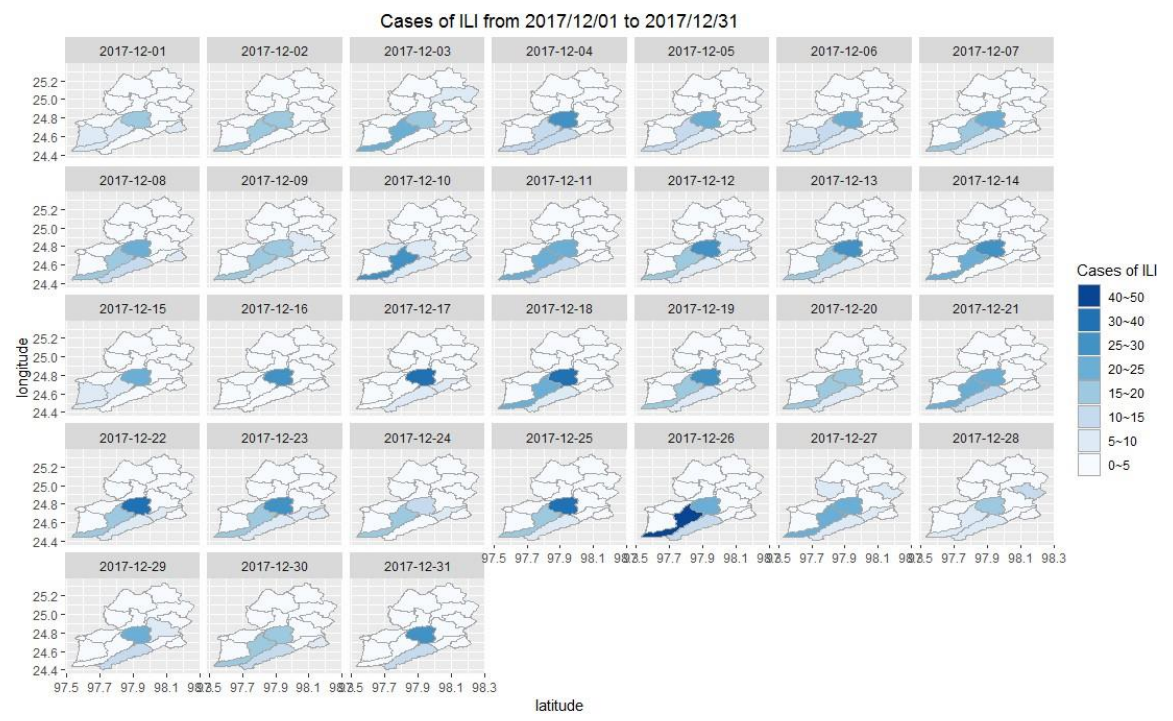

Figure 1. The spatio-temporal raw cases of ILI from 2017/12/01 to 2017/12/31

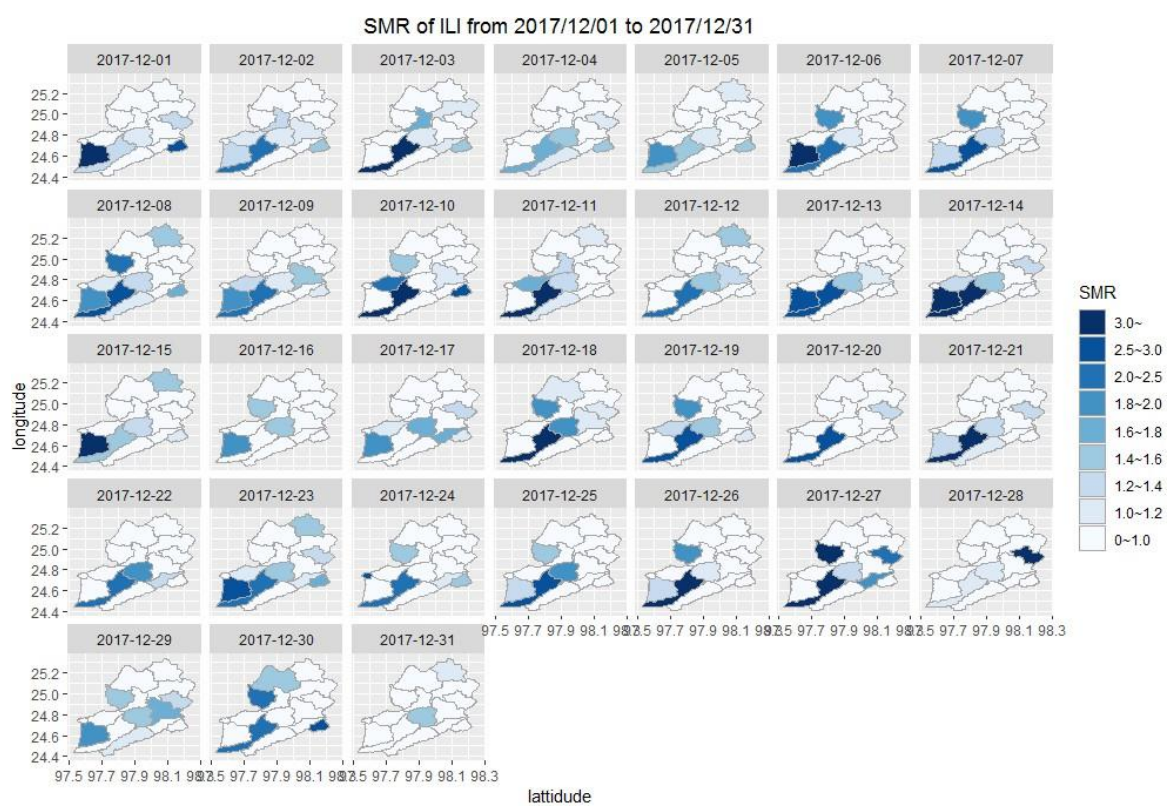

Figure 2. The SMR estimation risk of ILI 


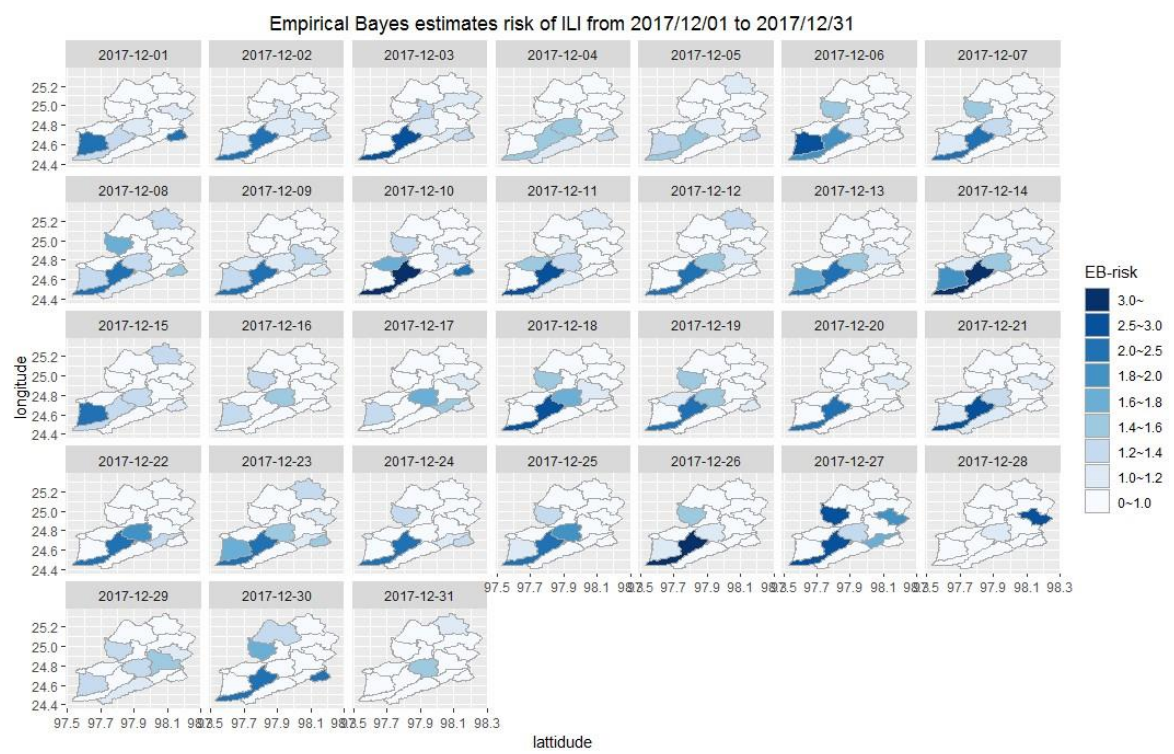

Figure 3. The EB-RR estimation risk of ILI

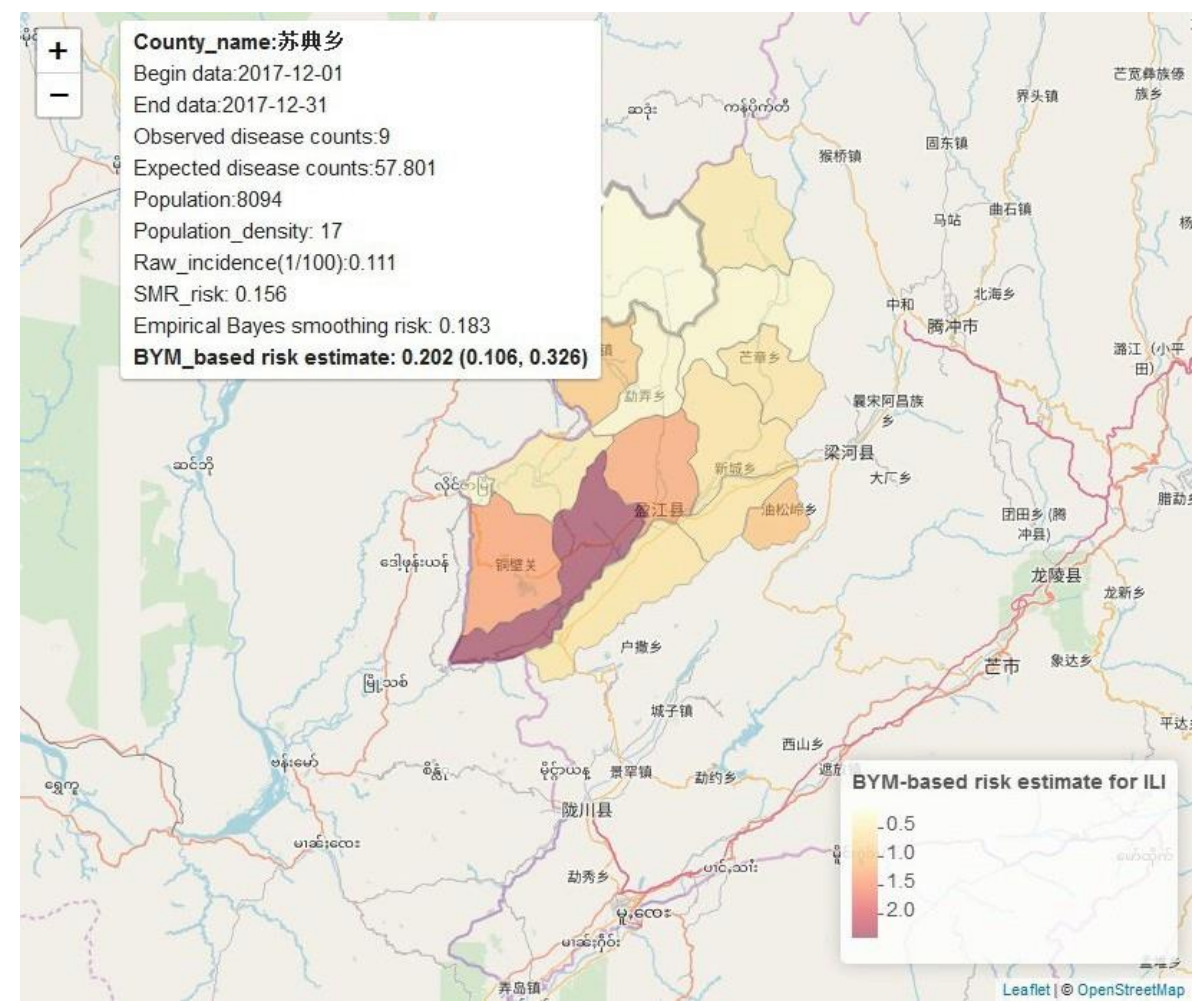

Figure 4. The screenshot of interactive visualization all the risk indexes 